\title{
EXTREME PERMISSIVISM REVISITED
}

\author{
Tamaz Tokhadze ${ }^{1}$ \\ ${ }^{1}$ University of Sussex \\ Original scientific article - Received: 23/09/2021 Accepted: 04/12/2021
}

\begin{abstract}
Extreme Permissivism is the view that a body of evidence could rationally permit both the attitude of belief and disbelief towards a proposition. This paper puts forward a new argument against Extreme Permissivism, which improves on a similar style of argument due to Roger White $(2005,2014)$. White's argument is built around the principle that the support relation between evidence and a hypothesis is objective: so that if evidence $E$ makes it rational for an agent to believe a hypothesis $H$, then $E$ makes it rational to believe $H$, for all agents. In this paper, I construct a new argument against Extreme Permissivism that appeals to a logically weaker, less demanding view about evidential support, Relational Objectivity: whether a body of evidence $E$ is more likely if $H$ is true than if $H$ is false is an objective matter and does not depend on how any agent interprets the relationship between $E$ and $H$. Relational Objectivity is solely concerned with the conditional probabilities called likelihoods and does not put substantive constraints on an agent's prior and posterior credences. For this reason, the presented argument avoids the standard permissivist criticism levelled against White's argument.
\end{abstract}

Keywords: permissivism; uniqueness; Roger White; objectivity of evidential support; relational objectivity; epistemic standards; likelihoods 


\section{Introduction}

Can a body of evidence equally justify both believing a proposition and its negation? According to the view called Extreme Permissivism, the answer is yes. More fully:

Extreme Permissivism (EP): There are some bodies of evidence $E$, such that $E$ rationally permits believing that $H$ and believing that $\neg H$.

Roger White (2005, 2014), who has coined the term "Extreme Permissivism", has put forward several arguments against EP. At the heart of White's central arguments is the idea I call the Objectivity of Evidential Support (Objectivity, for short): the view that the support relation between evidence and a proposition (or hypothesis) is absolute and does not change from agent to agent. So, according to Objectivity, if the evidence supports a hypothesis, e.g. anthropogenic climate change, then the evidence supports the hypothesis for all agents. And given some plausible assumptions, Objectivity seems to entail that rational individuals cannot respond differently to the same evidence. ${ }^{1}$

Many permissivists have argued that White presupposes "a superseded view of evidential support" (Douven 2009, 347). According to this standard permissivist response, it is a mistake to view the support relation as a two-place relation between evidence and a hypothesis (or a proposition); instead, evidential support can be sensitive to various third, agent-relative factors, such as epistemic standards, personal credence functions, epistemic goals or cognitive abilities. ${ }^{2}$ For instance, according to this line of thought, equally informed jurors may come to different but equally justified conclusions about whether a defendant is guilty because they have different epistemic standards on what counts as sufficient and relevant evidence for the defendant's guilt.

In this paper, I offer a novel argument against EP that captures the plausible thought behind Objectivity and is immune to the popular permissivist objection that I've outlined above. My argument substitutes Objectivity with a less demanding thesis I call Relational Objectivity: whether a body

\footnotetext{
${ }^{1}$ A similar argument can be found in Feldman (2007) and Matheson (2011). All the other criticisms of EP that I'm aware of are committed to Objectivity: e.g. Hedden (2015), Dogramaci and Horowitz (2016), Greco and Hedden (2016), Stapleford (2019).

${ }^{2}$ The view that evidential support, at least in some cases, is agent-relative (or requires some agentrelative factor) has been defended by Douven (2009), Titelbaum (2010), Decker (2012), Kelly (2014), Meacham (2014), Peels and Booth (2014), Schoenfield (2014), Kopec and Titelbaum (2016, 2019), Podgorski (2016), Simpson (2017), Jackson (2019), Jackson and Turnbull (forthcoming).
} 
of evidence $E$ is more likely if a hypothesis, $H$, is true than if $H$ is false depends on $E$ and $H$ themselves and not on how any agent interprets the relationship between $E$ and $H$. As its name suggests, Relational Objectivity is a relational or contrastive principle. It is solely concerned with the conditional probabilities called likelihoods, and does not put substantive constraints on prior and posterior credences of an agent. For this reason, Relational Objectivity is wholly compatible with the view that there are important agent-relative factors that influence what an agent ought to believe and to what degree.

The paper proceeds as follows. After discussing some preliminaries in section 2, I introduce and analyse White's argument against EP in section 3. Discussing White's argument will allow us to see in what respects the novel argument against EP, introduced in section 4, departs from White's original argument. I conclude in section 5 that it is possible to reject EP and still retain an important permissivist idea that subjective, agent-relative factors rationally influence an agent's doxastic states. Hence, I submit that the proposed novel argument against EP improves on a similar style of argument due to White.

\section{Varieties of Permissivism}

There are many versions of Permissivism. In this paper, we are solely concerned with Permissivism about (categorical, coarse-grained) belief, which states that a body of evidence can equally justify both the attitude of belief and disbelief towards a proposition.

The arguments against EP discussed in this paper are compatible with weaker versions of Permissivism, such as Moderate Permissivism and Credal Permissivism: ${ }^{3}$

Moderate Permissivism: There are some bodies of evidence $E$, such that $E$ rationally permits two belief-attitudes towards a proposition, where suspension of judgment about the proposition is among the permitted attitudes.

Credal Permissivism: There are some bodies of evidence $E$, such that $E$ rationally permits more than one credence towards a proposition.

\footnotetext{
${ }^{3}$ See also Jackson (2019) for a discussion of various versions of Permissivism.
} 
Moderate Permissivism allows that in some cases, a body of evidence permits, say, belief that $H$ and suspending judgement about $H$. One may endorse Moderate Permissivism without endorsing EP. And while, as stated, EP does not logically imply Moderate Permissivism, it is plausible to assume that if EP is true, then Moderate Permissivism is also true. ${ }^{4}$

Regarding Credal Permissivism: it is a thesis about fine-grained doxastic attitudes and does not imply any similar thesis about the coarse-grained attitude of belief. While EP is a thesis about (coarse-grained) belief, it implies Credal Permissivism on a widely accepted assumption that one should believe a proposition only if one assigns a high credence to that proposition.

Credal Permissivism is an extremely popular position within contemporary epistemology; ${ }^{5}$ and for that reason alone, it should be considered vastly more plausible than EP.

The negation of Permissivism is called Uniqueness. As with Permissivism, Uniqueness comes in many forms. Because this paper criticises EP, it advocates the view I call Moderate Uniqueness (the negation of EP):

Moderate Uniqueness: For any hypothesis $H$ and evidence $E$, it is not the case that $E$ justifies/rationally permits belief that $H$ and belief that $\neg H{ }^{6}$

Now that all the key terms have been defined, we are ready to state and analyse White's argument against EP.

\section{White's Argument against EP}

As noted in the introduction, the key premise in White's argument is Objectivity: the view that the support relation between evidence and a proposition (or hypothesis) is absolute and does not change from agent to agent. White (2014) has specified Objectivity in modal terms, as the thesis

\footnotetext{
${ }^{4}$ We could have stated EP as the thesis that some bodies of evidence equally justify all three beliefattitudes towards a proposition: belief, disbelief and suspension. But as we are solely focused on the permissibility of adopting opposing beliefs towards a proposition, it is unnecessary to strengthen EP.

${ }^{5}$ To quote Douven $(2009,348)$ “ (...) to the best of my knowledge no one calling him- or herself a Bayesian thinks that we could reasonably impose additional constraints that would fix a unique degrees-of-belief function to be adopted by any rational person". Douven is completely right. Even contemporary Objective Bayesians, such as Williamson (2010), grant that some evidential situations permit more than one credence towards a hypothesis.

${ }^{6}$ I will use the terms "justified" and "rational" interchangeably. While in other contexts, the two notions could be distinguished, such a distinction would serve no useful purpose in this paper.
} 
that evidential support relations hold necessarily: that is, if $E$ supports $H$ then necessarily $E$ supports $H$.

The claim that the evidential support relation holds necessarily may sound unobvious, and even trivially false to some. To take White's (ibid., 313314) example that illustrates the worry about the necessity claim:

That the gas gauge reads Full supports the conclusion that the tank is full. But it need not. Suppose we know that the gauge is stuck on Full, or even that the wiring is switched so that it tends to read Full only when the tank is empty. In these cases the gauge's reading Full seems to support no conclusion or the opposite conclusion.

So, according to the above example, the evidence $g$ : "The gas gauge reads Full" may support different conclusions, depending on what else we know about the gas gauge. To this example, White responds that $g$, in itself, does not support any conclusion about the tank. It is only when we combine $g$ with our background evidence that we can meaningfully talk about what the evidence supports. For instance, if our background evidence is that the gauge is typically reliable, then $g$ unequivocally supports the conclusion that the tank is full. As White (ibid., 314) concludes, when our background evidence is sufficiently specified, "it is hard to make sense of the idea that all of that information might have supported a different conclusion".

Now, using some additional premises, White gives the following argument against EP from Objectivity $(2014,314):^{7}$

\section{The Evidential Support Argument}

(P1) If $E$ supports $H$ then necessarily $E$ supports $H$.

(P2) It cannot be that $E$ supports $H$ and $E$ supports $\neg H$.

(P3) Necessarily, it is rational for $S$ to believe that $H$ iff $S$ 's total evidence supports $H$.

Therefore:

(C1) If an agent whose total evidence is $E$ is rational in believing $H$, then it is impossible for an agent with total evidence $E$ to rationally believe $\neg H$.

\footnotetext{
${ }^{7}$ The argument is quoted verbatim, but the order of premises and the original formalism is changed for the uniformity of reading.
} 
The first premise is White's version of Objectivity. Regarding the two other premises: P2 is what might be called the Univocity Principle (Univocity, for short), the view that "evidence speaks univocally, not equivocally" (Weisberg 2020, 2). So, according to Univocity, if evidence points to $H$ it cannot also point to $\neg H$. The last premise, P3, is a bridge principle connecting evidential support with justified/rational belief. And the conclusion of White's argument, $\mathrm{C} 1$, is equivalent to Moderate Uniqueness (the negation of EP).

Permissivists have found the argument unconvincing. The most popular criticism of the argument is centred around White's account of evidential support. Several authors have argued that the relation of support is always relative to a third relatum. To quote Kopec and Titelbaum:

(...) support facts obtain only relative to a third relatum; absent the specification of that third relatum, there simply is no matter of fact about whether the evidence justifies the hypothesis. (Kopec and Titelbaum 2019, 208)

Permissivists have developed a couple of different interpretations of this "third relatum" (see Kopec and Titelbaum 2016, 194); the most common interpretation is in terms of epistemic standards (Schoenfield 2014). Epistemic standards are the norms of evaluating and reasoning about evidence deemed reliable or truth conducive.

A popular and elegant way of representing epistemic standards is in terms of Bayesian credence functions. According to the standard Bayesian position, the degree to which an agent ought to believe a hypothesis, $H$, depends on (at least) two factors: (i) her (total body of) evidence and (ii) her prior probability in $H$. Prior probabilities (or priors) encode an agent's degree of belief in $H$ before receiving evidence $E$. An agent's priors may reflect her epistemic standards: say, how much an agent values the simplicity of a hypothesis compared to its explanatory power. So, equally rational agents may adopt non-trivially different priors, depending on how much weight they give to the simplicity considerations over the explanatory considerations; and different priors may lead to non-trivially different posteriors.

Hence, permissivists contend that two individuals can rationally respond to the same body of evidence differently if they endorse different epistemic standards.

In the next section, I'll state a novel argument against EP which avoids the standard criticism of White's argument. I will substitute White's 
Objectivity with a relatively undemanding principle about evidential support, which I call Relational Objectivity: the view that whether a body of evidence is more likely if a hypothesis is true than if the hypothesis is false is an objective matter. On White's argument, the objective support relation has the belief-guiding role (see the third premise, $\mathrm{P} 3$; more on this in the next section). By contrast, the presented argument won't assume that relational facts about support (fully) determine what an agent should believe and to what degree. For this reason, Relational Objectivity won't be susceptible to the standard permissivist objections; or so I will argue.

\section{A New Argument against EP}

My argument against EP consists of three premises and a theorem of the probability theory. The first premise is a conditional that states that EP implies the existence of a certain type of permissive cases, and the other two premises are epistemic principles which I call the Moderate Principle, and Relational Objectivity. In what follows, first, I'll state the argument in a premise-conclusion form and then discuss these premises one at a time.

The Relational Objectivity Argument

(1) If EP is true, then two equally informed agents who rationally suspend judgment about $H$ can rationally come to adopt opposing doxastic attitudes about $H$ upon learning some new evidence $E$ : one agent may believe $H$ and the other agent $\neg H$.

(2) The Moderate Principle: If evidence $E$ justifies you in believing that $H$ and prior to learning that $E$, you were not justified in believing $H$, then $E$ makes it rational to increase your probability in $H$; i.e., $P(H \mid E)>P(H)$, where $P$ represents your credence function and $P$ is a rational credence function for you to have.

(3) Relational Objectivity: Whether evidence $E$ is more likely on $H$ than on $\neg H$, depends on the evidence and hypotheses themselves and not on how any agent interprets the relationship between the evidence and hypotheses; i.e., for any two equally informed agents with rational credence functions $P$ and $P^{*}$, it cannot be the case that $P(E \mid H)>$ $P(E \mid \neg H)$ and $P^{*}(E \mid H) \leq P^{*}(E \mid \neg H)$. 
(4) Theorem: For any $H, E$ and credence function $P, E$ confirms $H$ iff $P(E \mid H)>P(E \mid \neg H){ }^{8}$

Therefore:

(5) Moderate Uniqueness: For any hypothesis $H$ and evidence $E$, it is not the case that $E$ justifies belief that $H$ and belief that $\neg H$.

The argument is valid. To see this, assume for reductio that EP is true. Given the first premise and the Moderate Principle, EP entails that a body of evidence $E$ could confirm $H$ for one agent and $\neg H$ for some other agent. Now, it is a theorem of the probability theory, that, for any $H$ and probability function $P, E$ confirms $H$ iff $P(E \mid H)>P(E \mid \neg H)$. And by Relational Objectivity, if the inequality $P(E \mid H)>P(E \mid \neg H)$ is true for some agent, then it is true for all equally informed agents. Therefore, it cannot be the case that $E$ confirms $H$ for one agent and $\neg H$ for some other agent; contrary to our assumption.

Now that we have established the argument's validity, let us proceed to discuss each of its premises, one at a time.

The first premise does not follow from the definition of EP, but it articulates the key idea behind EP; that some bodies of evidence, in themselves, are radically permissive: so the reason why two individuals can adopt opposing doxastic attitudes towards $H$ in light of their shared evidence is not because of their prior convictions about $H$ but because of their different evaluation of the same evidence. ${ }^{9}$ Let me provide an example: consider two open-minded agents who, at some time, share the same (background) evidence $K$ about the existence of God of traditional theism, $G$, and these agents rationally suspend judgment on $G$, in light of $K$. Now, according to premise (1), if EP is true, it may be possible that upon learning some new evidence $E$ these agents rationally come to opposing conclusions about God's existence; so that one agent rationally believes $G$, while the other rationally believes $\neg G$.

I should note that premise (1) does not imply any substantive constraints on an agent's degrees of belief or credences. If two agents are agnostic

\footnotetext{
${ }^{8} \mathrm{I}$ always assume that for all hypotheses $x, 0<P(x)<1$.

${ }^{9}$ Many permissivists like Kelly (2014), Schoenfield (2014), and Kopec and Titelbaum (2019) explicitly argue that Permissivism is true because rational individuals can evaluate the same evidence in different ways, and not because they already have opposing attitudes towards $H$ without any evidence. See, for instance, Kopec and Titelbaum's (2019., Sect. 4) Reasoning Room example. So given the published defences of EP, a version of EP that is incompatible with premise (1) does not seem to be an appealing view even for permissivists.
} 
about God's existence, this does not imply that their credences in God are the same. For instance, two agents may agree that a necessary condition for believing $G$ is that it has a high probability of being true, say higher than 0.7. So, on this assumption, both agents may suspend judgment on $G$, even if one is, say, 0.6 confident in $G$ and the other is 0.4 confident in $G$. As premise (1) is highly plausible, I expect that it won't be a controversial step in my argument.

The second premise, the Moderate Principle, as its name suggests, is a moderate, uncontroversial thesis. It does not say that you are rational in believing $H$ based on $E$ whenever $E$ rationally increases your probability or rationally confirms $H$. It only states a necessary (and not sufficient) condition on when it is rational to start believing $H$ based on $E$; and this necessary condition is that $E$ rationally confirms $H$. For instance, let $H$ be the hypothesis that Smith did the crime, let $E$ be some new body of evidence; say, the evidence that Smith's fingerprints were found in the crime scene. Now, if prior to receiving evidence $E$ you were not rational in believing $H$, and if $E$ makes you rational in believing that $H$, then $E$ must, at least, make it rational to be more confident in $H$ than before. ${ }^{10}$

What makes an agent's credence function rational (or rationally permissible)? Subjective Bayesians hold that the only rationality requirement on an agent's credence function $P$ is that $P$ is a probability function (that is, $P$ satisfies the standard axioms of probability). This requirement is called coherence. As we will see, via premise (3), I'll defend an additional constraint on $P$ that goes beyond (probabilistic) coherence; ${ }^{11}$ but I won't appeal to any set of conditions that are jointly sufficient for $P$ to be rational (for an agent).

It is important to note that whether $E$ confirms $H$ for an agent depends on what else the agent knows or takes for granted. So it is useful to divide an

\footnotetext{
${ }^{10}$ One may object to the Moderate Principle for a reason related to the debate about the permissibility to form a belief in light of "mere statistical evidence". For instance, suppose that new evidence reduces your probability for a hypothesis but gives you non-statistical evidence for it, where you previously had only statistical evidence for it. Now, if we think that mere statistical evidence cannot suffice for rational belief, then we'll get cases where gaining evidence can justify moving from suspension of judgment to belief, despite reducing the probability of the believed proposition. Such alleged counterexamples against the Moderate Principle are irrelevant to the argument of this paper. Even if mere statistical evidence is insufficient for rational belief (which is a controversial assumption), we can restrict the argument against EP to the cases that do not involve a transfer from statistical to nonstatistical evidence. After all, there is no reason whatsoever to think that EP is only true when an agent's evidence changes from statistical to non-statistical evidence.

${ }^{11}$ Of course, it is not surprising that any argument against EP should go beyond a purely subjective Bayesian account of confirmation. I should also note that, while subjective Bayesianism is a popular view, many (e.g. see Maher 1996; Hawthorne 2005) have argued that any purely subjective account of confirmation faces some serious problems, most notably the so-called problem(s) of old evidence. See Maher (1996) for a detailed argument that a purely subjective account of confirmation is unattainable.
} 
agent's total body of evidence into two parts: new evidence $E$ and a body of background evidence, denoted by $K$. What counts as new evidence $E$ and what counts as background evidence $K$ is largely an arbitrary matter and depends on an agent in question and her context of reasoning. For instance, suppose you are particularly interested in how a piece of evidence $E$ bears on the hypothesis, $H$, that Smith did the crime. $E$ may be the evidence that Smith's fingerprints were found at the crime scene. In evaluating evidence $E$, your background evidence will include every relevant proposition that you take for granted at that time: such as common-sense propositions about how the world works (e.g. people leave fingerprints and that the fingerprint matching technology is highly accurate) and the assumption that the evidence has not been planted, etc. So given your background evidence $K$, it is clear that $E$ confirms $H$.

If we make an agent's background evidence explicit, ${ }^{12}$ the Moderate Principle can be stated more fully as follows (for simplicity, I assume that both $E$ and $K$ are sets of propositions):

The Moderate Principle: Suppose your total body of evidence is $E \cup K$. If evidence $E$ justifies you in believing that $H$ and prior to learning that $E, K$ alone did not justify you in believing $H$, then $P(H \mid E \wedge K)>P(H \mid K)$, where $P$ represents your credence function and $P$ is a rational credence function for you to have.

The Moderate Principle is as plausible as an abstract epistemic principle can be. It is neutral between permissivist and impermissivist epistemologies. For instance, within the subjective Bayesian framework, the Moderate Principle is obviously right: after all, if relative to your credence function $P$, a new piece of evidence $E$ does not increase your credence in $H$, then why start believing $H$ on $E$ ? If $E$ does not add to your credence in $H$, then $E$ cannot be a part of your reason for believing $H$.

Therefore, I also do not expect the Moderate Principle to be a controversial premise in my argument.

By contrast, the third premise, Relational Objectivity, is a controversial premise from a permissivist perspective. So, it requires a more detailed discussion and motivation, compared to the previous two premises.

\footnotetext{
${ }^{12}$ Sometimes, for the sake of readability, I won't explicitly mention an agent's background evidence. But it should be remembered that the talk of confirmation only makes sense relative to a given background evidence. I will make background evidence explicit only when necessary.
} 
Relational Objectivity is a comparative principle: it is solely concerned with how likely evidence $E$ is if a hypothesis $H$ is true than if $H$ is false. Relational Objectivity is not concerned with either a prior probability of a hypothesis $H$, denoted by $P(H)$, or a posterior probability of $H, P(H \mid E)$. Instead, Relational Objectivity is about the conditional probabilities of the following type- $P$ (evidence|hypothesis), called likelihoods. A likelihood encodes what a hypothesis, $H$, says about evidence $E$ : that is, how likely $E$ is on the supposition that $H$ is true.

As with the confirmation relation, whether $P(E \mid H)>P(E \mid \neg H)$ depends on a body of background evidence $K$. So, taking background evidence into account, Relational Objectivity can be stated more precisely as follows:

Let $P$ and $P^{*}$ be rational credence functions of two agents who share the same background evidence $K$; then for any evidence $E$ it cannot be the case that $P(E \mid H \wedge K)>P(E \mid \neg H \wedge K)$ and $P^{*}(E \mid H \wedge K) \leq P^{*}(E \mid \neg H \wedge K)$.

The relevance of Relational Objectivity for our argument is made explicit by the theorem of probability theory:

Theorem: For any $H, E, K$, and probability function $P, E$ confirms $H$ relative to $K$ iff $P(E \mid H \wedge K)>P(E \mid \neg H \wedge K)$.

So, given this theorem, Relational Objectivity is equivalent to the thesis that if a piece of evidence $E$ (relative to the fixed background evidence) rationally confirms a hypothesis, then it rationally confirms the hypothesis for all (equally informed) agents.

It may be useful to note that, instead of Relational Objectivity, I could have used a similar principle that has been defended by Maher (1996, 163). Maher has argued that the following, more objectivist analysis of confirmation should substitute the subjective Bayesian analysis of confirmation:

Let $R(K)$ denote the set of all probability functions that are rationally permissible on background evidence $K$; then $E$ confirms $H$ relative to $K$, iff for all $P \in R(K), P(H \mid E \wedge K)>$ $P(H \mid K)$.

The gist of Relational Objectivity and Maher's principle is the same: on both principles, whether evidence $E$ rationally confirms $H$ does not depend on how an agent subjectively evaluates the relationship between $E$ 
and $H$ (relative to $K$ ). Clearly, my argument would remain valid if we substitute Relational Objectivity with Maher's principle.

But, unlike Maher's principle, Relational Objectivity makes explicit that the objectivity of confirmation is due to, what Hawthorne $(2005,278)$ has called "the objectivity or "publicness" of likelihoods that occur in Bayes" theorem" (More on this in section 4.1). So, Relational Objectivity is stated in a way that emphasises this publicness or objectivity of likelihoods.

Why accept Relational Objectivity? Firstly, Relational Objectivity is logically weaker than White's Objectivity: the former is entailed by the latter but not the other way around. So, any reason for accepting Objectivity is also a reason for accepting Relational Objectivity. Let me elaborate on this.

Objectivity is concerned with the traditional notion of evidential support which is closely related to the notion of rational belief. This is made explicit by the third premise of White's argument:

(P3) Necessarily, it is rational for $S$ to believe that $H$ iff $S$ 's total evidence supports $H$.

By contrast, as Theorem makes explicit, Relational Objectivity is concerned with the notion of confirmation. And the confirmatory relation between $E$ and $H$ is necessary but often insufficient for an agent to rationally believe $H$ on $E$, even if $S$ 's total body of evidence is $E$ (I give an example shortly). An alternative way of explaining the difference between White's Objectivity and Relational Objectivity is by invoking Carnap's (1962, Preface to the Second Edition) well-known distinction between "concepts of firmness" and "concepts of increase in firmness". White's Objectivity concerns the firmness of a hypothesis; it says that whether a hypothesis is sufficiently firm or probable (for belief) is an objective matter. By contrast, Relational Objective concerns whether the evidence increases the firmness or confirms the hypothesis. And, as it is well-known, the evidence may increase the firmness of a hypothesis without making the hypothesis firm (or sufficiently firm). For instance, consider a detective who received reliable testimony that a suspect, John, was seen near the crime scene. Suppose that this piece of evidence, $T$, is the detective's total body of evidence that Jonn committed the crime (denoted by $J$ ). Now, even if $T$ rationally confirms or increases the probability of $J$, it is clearly irrational to believe that $J$ solely on the basis of $T$. In Carnap's terms, $T$ increases the firmness of $J$ but does not make $J$ firm enough (for the detective). Hence, while confirmation is necessary for rational belief, it is often insufficient. 
To sum up: Relational Objectivity is motivated by the same core idea as Objectivity, that the evidential support relation is objective, at least to some extent. But, unlike White's Objectivity, Relational Objectivity only commits us to a moderate view about the extent to which the support relation is objective.

Certainly, permissivists may call Relational Objectivity into question. But, as we will see, to call Relational Objectivity into question requires more than the appeal to the standard permissivists claims: that subjective, agentrelative factors such as epistemic standards, goals, and personal credence functions have a rational influence on an agent's doxastic states. So, I'll be happy to concede to permissivists that there is no objective support relation in White's sense: where the objective support relation fully determines what an agent ought to believe. However, as I argue next, Relational Objectivity won't commit us to such a demanding view about objective support.

\subsection{Relational Objectivity}

As I've already explained, Relational Objectivity is solely concerned with the type of conditional probabilities called likelihoods. Unlike prior and posterior probabilities, likelihoods are widely considered to be the most objective part of Bayesian inference. To illustrate this, let us consider one of the most common forms of Bayes' theorem:

$$
\text { (1) } P(H \mid E)=\frac{P(E \mid H) * P(H)}{P(E \mid H) * P(H)+P(E \mid \neg H) * P(\neg H)}
$$

Equation (1) enables us to calculate posterior probability, $P(H \mid E)$, in terms of the prior probability of $H, P(H)$, and two likelihoods: $P(E \mid H)$ and $P(E \mid \neg H) \cdot{ }^{13}$ Notice that (by the law of total probability) the denominator in Bayes' theorem- $P(E \mid H) * P(H)+P(E \mid \neg H) * P(\neg H)$ - equals to the expectedness of evidence, $P(E)$. Hence, $P(E)$ is equivalent to the probability-weighted average of likelihoods. So, (1) can be simplified to:

$$
\text { (2) } P(H \mid E)=\frac{P(E \mid H) * P(H)}{P(E)}
$$

And by using equation (2), if we take the ratio of $P(H \mid E)$ and $P(\neg H \mid E)$ we get the ratio form of Bayes' theorem:

\footnotetext{
${ }^{13}$ Sometimes, likelihoods written as $P(E \mid \neg H)$ denote the likelihoods of a special kind known as catchall likelihoods. Catchall likelihoods are discussed at the end of this section.
} 


$$
\text { (3) } \frac{P(H \mid E)}{P(\neg H \mid E)}=\frac{P(E \mid H) * P(H) / P(E)}{P(E \mid \neg H) * P(\neg H) / P(E)}
$$

By simplifying, we get:

$$
\text { (4) } \frac{P(H \mid E)}{P(\neg H \mid E)}=\frac{P(E \mid H)}{P(E \mid \neg H)} * \frac{P(H)}{P(\neg H)}
$$

Finally, let $R_{\text {Post }}$ be the ratio of posteriors, $R_{L}$ the ratio of likelihoods, and $R_{\text {Prior }}$ the ratio of priors, then, the ratio form of Bayes' theorem can be summarised succinctly as:

$$
\text { (5) } R_{\text {Post }}=R_{L} * R_{\text {Prior }}
$$

As equation (5) makes explicit, the impact of evidence on any pair of priors is completely exhausted by $R_{L}$, the ratio of likelihoods. ${ }^{14}$

Relational Objectivity is solely concerned with the value of $R_{L}$ and not at all concerned with $R_{\text {Prior }}$ and $R_{\text {Post }}$. This is an important selling point of Relational Objectivity as prior probabilities are unanimously acknowledged as the most subjective and problematic part of Bayesian inference. And what makes Relational Objectivity more appealing is that it is not a quantitative but a comparative principle: Relational Objectivity is not concerned with the precise numerical values of likelihoods, but only with their comparative probabilities. As $R_{L}=P(E \mid H) / P(E \mid \neg H)$, it follows that $P(E \mid H)>P(E \mid \neg H)$ iff $R_{L}>1$. So, on Relational Objectivity, the exact value of $R_{L}$ is unimportant; what is important is whether $R_{L}$ is greater than 1 .

Now, even the so-called subjective Bayesians - that is, Bayesians who allow the multitude of coherent prior distributions as rationally permissible -accept that $R_{L}$ is the most objective part of Bayesian inference (Hawthorne 2005, 283). The objective status of $R_{L}$ is due to the fact that, in many cases, an agent's evidence defines an objective (or intersubjectively justified) probability distribution over a set of competing

\footnotetext{
${ }^{14}$ The claim that the ratio of likelihoods is the only factor that impacts how the evidence changes the ratio of priors (which, as equation (5) illustrates, is a fact of probability theory) should not be conflated with a different claim that the ratio of likelihoods provides the adequate measure of the degree to which the evidence confirms a hypothesis. While some have argued that likelihoods are sufficient to adequately measure confirmation, not everyone accepts this. See Festa and Cevolani (2017) for a relevant discussion and references.
} 
hypotheses without presupposing any prior probability distribution over these hypotheses. ${ }^{15}$

To illustrate the independence of likelihoods from prior probabilities, consider the following diagnostic example (a more philosophical example is considered shortly):

You are a physician who assesses a patient on whether she has some skin disease $D$. Based on the extensive medical records, you know that the symptoms $S$, a peculiar rash on her hands, is $90 \%$ likely if she has $D$ and only $10 \%$ likely if she does not have $D$. So, you know that $P(S \mid D)=0.9>P(S \mid \neg D)=0.1$.

Based on this information, you already know that evidence $S$ confirms $D$ : $P(D \mid S)>P(D)$. And as the likelihood ratio is quite high, $P(S \mid D) /$ $P(S \mid \neg D)=0.9 / 0.1=9$, we know that the evidence $S$ makes the posterior ratio, $P(D \mid S) / P(\neg D \mid S)$, nine times greater than the prior ratio, $P(D) / P(\neg D)$. So it is clear that $S$ provides quite good evidence for $D$. But, this being said, the new evidence, $S$, is insufficient to conclude that the posterior probability of $D$ is high (say, higher than 0.5 ). This is so, because the prior of $D$ may be quite low. So, suppose that $D$ is a rare disease and only 1 in 1000 have it. And if your prior in $D$ is 1/1000, then, unintuitively, simple calculations show that your posterior probability in $D$ should be less than $1 \%: P(D \mid S) \approx 0.009$. And in general, even if you do not have sufficient information to provide an objective, uncontentious estimate of the prior of $D$, you can still rationally conclude that $S$ is more likely on $D$ than on $\neg D$ : hence you can rationally conclude that $S$ confirms $D$.

As this diagnostic example illustrates, likelihoods may be independent of priors and in many contexts have "objective or inter-subjectively agreed values" (Hawthorne 2005, 283). For this reason, even subjective Bayesians accept the special status of likelihoods; for instance, Edwards et al. (1963, 199) called likelihoods public because "[i]n many applications practically all concerned find themselves in substantial agreement with regard to [likelihoods]".

Certainly, fixing the precise numerical values of likelihoods is not always as easy and objective as in the above diagnostic example. However, Relational Objectivity is not concerned with precise numerical values of likelihoods but merely with their comparative plausibilities. So, on Relational Objectivity, whether $P(E \mid H)$ is greater than $P(E \mid \neg H)$ is an

\footnotetext{
${ }^{15}$ See also Bandyopadhyay et al. (2016) for a detailed discussion about the special status and role of likelihoods in Bayesian inference.
} 
objective matter, even if $P(E \mid H)$ and $P(E \mid \neg H)$ do not always have objective numerical values. This makes Relational Objectivity a modest and appealing thesis even from a subjectivist perspective; as there are many cases where the exact numerical values of likelihoods are highly debatable, but we may still be in a position to know comparative claims about these likelihoods. To illustrate this, consider the following, more philosophically interesting example. Suppose two agents agree that the existence of evil constitutes evidence against God's existence, in the sense that the existence of evil is less likely if God exists than if God does not exist: $P($ evil exists $\mid$ God $)<P($ evil exists $\mid$ no God $)$. And the agreement about the comparative plausibilities of these likelihoods requires neither the agreement about the priors, nor the agreement about the precise numerical values of these likelihoods.

While in many scientific and philosophical settings comparative claims about likelihoods are objective (or intersubjectively justified), it is unrealistic to suppose that this is always the case. Essentially, the problem is that sometimes it is not possible to approximate in a non-subjective manner the values of the so-called catchall likelihoods: the likelihoods that contain catchall (or composite) hypotheses. A catchall hypothesis is a disjunction of simple (or non-composite) hypotheses. To take an easy example: the hypothesis $H_{1}$ : "the coin is fair" is simple while the hypothesis $\neg H_{1}$ : "the coin is not fair" is a catchall, as $\neg H_{1}$ is the disjunction of all the specific alternatives to $H_{1}$ (there are many specific ways in which the coin fails to be fair, if not assumed otherwise). Now, suppose the coin is tossed ten times and eight heads are obtained (denote this observation as " $e$ "). The likelihood of $e$ on the supposition that the coin is fair, $H_{1}$, is completely objective and does not require the specification of a prior distribution over the competing hypotheses (no matter what the prior distribution is, $\left.P\left(e \mid H_{1}\right)=45 * 0.5^{10} \approx 0.04\right)$. By contrast, the corresponding catchall likelihood, $P\left(e \mid \neg H_{1}\right)$ is sensitive to the prior distribution. To calculate the value of $P\left(e \mid \neg H_{1}\right)$ we must know the values of all ordinary likelihoods of $e$ on each specific (mutually exclusive) alternative to $H_{1}$ and the prior distribution over these alternatives. In symbols:

$$
P\left(e \mid \neg H_{1}\right)=\frac{\sum_{i \neq 1} P\left(e \mid H_{i}\right) * P\left(H_{i}\right)}{P\left(\neg H_{1}\right)}
$$

So, mathematically, catchall likelihoods are reducible to priors and ordinary likelihoods: if we know the values of priors and likelihoods, then we can calculate the value of any catchall likelihood. Thus, in many important settings, the values of catchall likelihoods cannot be neatly 
separated from the values of priors. The reader may worry that this feature of catchall likelihoods calls the argument of this paper into question, as the argument relies on the independence of likelihoods and priors.

But the sensitivity of catchall likelihoods on priors is wholly consistent with my argument. For a start, there is an important asymmetry between catchall likelihoods and priors (Fitelson 2007, Section 5). If one knows the values of priors and ordinary likelihoods, then one can calculate the value of the corresponding catchall likelihoods; but not the other way around. Knowing the value of catchall likelihoods and ordinary likelihoods does not determine the prior distribution. ${ }^{16}$ So, less information is required to determine the values of catchalls than to determine the values of priors. Because of this, the subjectivity of priors does not necessarily translate to the subjectivity of catchall likelihoods: we could have an objective approximation of the value of a catchall likelihood, but not the value of the corresponding priors.

I expect the following objection at this point: "But what if two equally rational agents have different estimates of priors and, due to this, they disagree about comparative claims involving catchall likelihoods? Does not this show that Relational Objectivity is false?" The answer is "No". Relational Objectivity does not entail a strong and unobvious claim that the inequalities between likelihoods are always objectively well-defined. It is compatible with Relational Objectivity that in some cases, the available evidence does not (objectively) justify even comparative claims about likelihoods (or confirmation). And if such cases obtain, i.e., if the available evidence does not justify comparative claims about likelihoods, then, according to my argument against EP, it is irrational to believe (or disbelieve) the relevant hypothesis, $H$, on that evidence. ${ }^{17}$ Instead, we should conclude that there is no fact of the matter whether $E$ is more likely on $H$ than on $\neg H$. This conclusion is entirely compatible with Relational Objectivity, as it does not require that the inequalities between likelihoods are objectively well-defined on any evidence.

Hopefully, this discussion convinces even those sympathetic with permissivist epistemologies that Relational Objectivity is a plausible,

\footnotetext{
${ }^{16}$ To take the coin example again, we could be justified to think that the likelihood of getting eight heads out of ten tosses is higher on the supposition that coin is not fair than on the supposition that the coin is fair, even if it is not possible to estimate the value of priors in a non-subjective manner.

${ }^{17}$ For instance, Sober $(2008,26-30)$ has argued that when we deal with "deep and general" theories, such as the general theory of relativity, then some comparative claims about likelihoods cannot be objectively justified. See Sober (Chapter 1, Sections 1.2, 1.3) for a detailed discussion. Sober's overall view agrees with our conclusion that if evidence and hypotheses do not justify the comparative claims about likelihoods, then an agent should abstain from forming beliefs on such evidence.
} 
moderate principle, especially compared to such principles as White's Objectivity that imposes very strong constraints on rational belief.

In the next and final section, I summarise the key points of the paper and conclude that the presented argument against EP improves upon White's similar style of argument.

\section{Conclusion}

I've presented a novel argument against Extreme Permissivism (EP). According to this argument, EP is false because two equally informed agents who suspend judgement about a proposition $H$ cannot adopt opposing attitudes towards $H$ upon receiving the same new evidence; as adopting opposing attitudes towards $H$ requires that $E$ confirms $H$ for one agent, but $\neg H$ for the other agent; but given a plausible and relatively weak principle about likelihoods - which I've called Relational Objectivitythis cannot be the case.

Since Relational Objectivity is only concerned with relational probabilities of likelihoods, the presented argument against EP is wholly compatible with a plausible permissivist idea that non-evidential factors - such as epistemic standards, goals, and credence functions - have (some) rational influence on what an agent ought to believe (and to what degree).

To illustrate this, suppose that Credal Permissivism is true (that is, equally informed agents can adopt different credences toward a proposition). Now, consider two equally informed detectives, Salome and Naomi, who adopt non-identical credence functions $P_{S}$ and $P_{N}$ and suspend judgment on whether John committed the crime (denoted by $J$ ). Salome may be more sceptical about John's guilt and attach lower prior in $J$; for simplicity, assume that relative to their shared background evidence $K, P_{S}(J)=0.25$ and $P_{N}(J)=0.4$. Now, further suppose that they learn a new piece of evidence $E$ that rationally confirms $J: P(E \mid J)>P(E \mid \neg J)$. Since Salome had a lower prior in $J$, her posterior in $J, P_{S}(J \mid E)$, may not be high enough for her to believe that $J$. By contrast, from Naomi's point of view, $P_{N}(J \mid E)$ could be sufficiently high to believe that $J$.

This example is wholly consistent with the presented argument against EP. So, even if Relational Objectivity is true, two agents may have nontrivially differing credences towards a proposition and rationally disagree about whether to believe or suspend judgment on that proposition. 
Certainly, supporters of EP may find Relational Objectivity too demanding and advance some novel objections against it. But, as I've argued, these objections must go beyond the standard permissivist claims that subjective, non-evidential factors have a rational influence on what an agent ought to believe and to what degree.

\section{Acknowledgments}

I am very grateful to Tony Booth, Corine Besson, Elizabeth Jackson, and JiMin Kwon for helpful comments and discussion on different parts and versions of this paper. Special thanks to Nina Abesadze for helpful discussions and feedback that shaped the argument of this paper. I'm also very grateful to two anonymous referees for this journal for challenging me to clarify and improve some important aspects of the paper.

\section{REFERENCES}

Bandyopadhyay, Prasanta S., Gordon Jr. Brittan, and Mark L. Taper. 2016. Belief, Evidence, and Uncertainty: Problems of Epistemic Inference. Springer International Publishing.

Carnap, Rudolf. 1962. Logical Foundations of Probability. 2nd. Chicago: University of Chicago Press.

Decker, Jason. 2012. "Disagreement, Evidence, and Agnosticism." Synthese 187: 753-783. https://doi.org/10.1007/s11229-0110010-x.

Dogramaci, Sinan, and Sophie Horowitz. 2016. "An Argument for Uniqueness about Evidential Support." Philosophical Issues 26 (1): 130-147. https://doi.org/10.1111/phis.12078.

Douven, Igor. 2009. "Uniqueness Revisited." American Philosophical Quarterly 46: 347-61.

Edwards, Ward, Harold Lindman, and Leonard J. Savage. 1963, 70. "Bayesian Statistical Inference for Psychological Research." Psychological Review: 193-242.

Feldman, Richard. 2007. "Reasonable Religious Disagreement." In Philosophers without Gods: Meditations on Atheism and the Secular Life, edited by Louise Antony, 194-214.

Festa, Roberto, and Gustavo Cevolani. 2017. "Unfolding the Grammar of Bayesian Confirmation: Likelihood and Antilikelihood Principles." Philosophy of Science 84 (1): 56-81. https://doi.org/10.1086/688935.

Fitelson, Branden. 2007. "Likelihoodism, Bayesianism, and Relational Confirmation." Synthese 156: 473-489. 
https://doi.org/10.1007/s11229-006-9134-9.

Greco, Daniel, and Brian Hedden. 2016. "Uniqueness and Metaepistemology." Journal of Philosophy 113 (8): 365-395.

Hawthorne, James. 2005. "Degree-of-Belief and Degree-of-Support: Why Bayesians need Both Notions.” Mind 114 (454): 277-320. https://doi.org/10.1093/mind/fzi277.

Hedden, Brian. 2015. "A Defense of Objectivism about Evidential Support." Canadian Journal of Philosophy 45 (5-6): 716-743. https://doi.org/10.1080/00455091.2015.1123038.

Jackson, Elizabeth. 2019. "A Defense of Intrapersonal Belief Permissivism." Episteme 1-15.

https://doi.org/10.1017/epi.2019.19.

Jackson, Elizabeth, and Margaret Greta Turnbull. Forthcoming. "Permissivism, Underdetermination, and Evidence." In The Routledge Handbook of the Philosophy of Evidence, edited by Clayton Littlejohn and Maria Lasonen-Aarnio.

Kelly, Thomas. 2014. "Evidence can be Permissive." In Contemporary Debates in Epistemology, edited by Matthias Steup, John Turri and Ernest Sosa, 298-312. Wiley Blackwell.

Kopec, Matthew, and Michael G. Titelbaum. 2016. "The Uniqueness Thesis." Philosophy Compass 11: 189-200.

https://doi.org/10.1111/phc3.12318.

Kopec, Matthew, and Michael G. Titelbaum. 2019. "When Rational Reasoners Reason Differently." In Reasoning: Essays on Theoretical and Practical Thinking, edited by Magdalena Balcerak Jackson and Jackson Balcerak-Jackson, 205-231. Oxford: Oxford University Press.

Maher, Patrick. 1996. "Subjective and Objective Confirmation." Philosophy of Science 63 (2): 149-174.

Matheson, Jonathan. 2011. "The Case for Rational Uniqueness." Logos \& Episteme 2 (3): 359-373.

https://doi.org/10.5840/logos-episteme20112319.

Meacham, Christopher. 2014. "Impermissive Bayesianism." Erkenntnis 79: 1185-217.

Peels, Rik, and Anthony Booth. 2014. "Why Responsible Belief is Permissible Belief." Analytic Philosophy 55: 75-88. https://doi.org/10.1111/phib.12036.

Podgorski, Abelard. 2016. "Dynamic Permissivism." Philosophical Studies 173 (7): 1923-1939. https://doi.org/10.1007/s11098-0150585-z.

Schoenfield, Miriam. 2014. "Permission to Believe: Why Permissivism is True and What it Tells us About Irrelevant Influences on Belief." Noûs 48: 193-218. https://doi.org/10.1111/nous.12006. 
Simpson, Robert. 2017. "Permissivism and the Arbitrariness Objection." Episteme 14 (4): 519-538. https://doi.org/10.1017/epi.2016.35.

Sober, Elliott. 2008. Evidence and Evolution: The Logic behind the Science. Cambridge University Press.

Stapleford, Scott. 2019. "Intraspecies Impermissivism.” Episteme 16 (3): 340-356. https://doi.org/10.1017/epi.2018.14.

Titelbaum, Michael G. 2010. "Not Enough There There: Evidence, Reasons, and Language Independence." Philosophical Perspectives 24 (1): 477-528.

Weisberg, Jonathan. 2020. "Could've Thought Otherwise." Philosophers' Imprint 20 (12): 1-24.

http://hdl.handle.net/2027/spo.3521354.0020.012.

White, Roger. 2005. "Epistemic Permissiveness." Philosophical Perspectives 19: 445-459. https://doi.org/10.1111/j.1520-8583.2005.00069.x.

White, Roger. 2014. "Evidence cannot be Permissive." In Epistemology, Contemporary Debates, edited by Matthias Steup, John Turri and Ernest Sosa, 312-323. Wiley-Blackwell.

Williamson, Jon. 2010. In Defence of Objective Bayesianism. Oxford University Press.

https://doi.org/10.1093/acprof:oso/9780199228003.001.0001. 
